\title{
Influence of the Interlayer Space on the Water Oxidation Performance in a Family of Surfactant-intercalated NiFe-Layered Double Hydroxides
}

\author{
Jose A. Carrasco, ${ }^{\ddagger}$ Roger Sanchis-Gual, ${ }^{\ddagger}$ Alvaro Seijas-Da Silva, Gonzalo Abellán, ${ }^{*}$ Eugenio Coro- \\ nado*
}

Instituto de Ciencia Molecular (ICMol), Universitat de València, Catedrático José Beltrán 2, 46980, Paterna, Spain.

\begin{abstract}
Layered double hydroxides (LDHs) are low dimensional materials that act as benchmark catalysts for the oxygen evolution reaction (OER). Many LDH properties affecting the OER have been studied to reach the optimal efficiency but no systematic studies concerning the influence of the interlayer space have been developed. In this context, these materials allow a large tunability in their chemical composition enabling the substitution of the interlayer anion and therefore modifying exclusively the basal space. Here, we synthesize by anion exchange reactions a surfactantintercalated family of NiFe-LDHs with increasing basal spacing ranging from 8.0 to $31.6 \AA$ (one of the largest reported so far for a NiFe-LDH) while the electrochemical OER performance of this family of compounds was explored to analyse the interlayer distance effect keeping similar morphology, dimensions and metallic composition. Results show the increase of the LDH basal space undergo to lower Tafel slopes, higher electrochemical surface area and a reduction of the resistance related to the chemisorption of oxygen leading to better kinetic behaviour, showing an optimum enhancement of the electrocatalytic performance for the NiFe-dodecyl sulphate (basal space of $25 \AA$ ). Interestingly, the NiFe-dodecyl sulphate exhibits optimum proton diffusion values, indeed a further increment in the basal space compromises the onset potential, a fact that could be related to an increase in the hydrophobicity between the layers. Moreover, by judicious tuning of the interlayer space, it is possible to reach a Tafel slope value for the most spaced LDH (NiFe-octadecyl sulphate, basal space of $31.6 \AA$ ), similar to the one obtained for exfoliated NiFe nanosheets, showing a much better long-time stability due to the three-dimensional robustness of the catalysts. This work illustrates the importance of molecular engineering in the design of novel highly active catalysts and provides important insights into the understanding of basic principles of oxygen evolution reaction in NiFe-LDHs.
\end{abstract}

\section{INTRODUCTION}

With the increasing requirements of reliable and environmentally friendly energy resources, electrochemical energy storage and conversion systems (such as supercapacitors and water splitting catalysts) have gathered intense interest in the last years. In this context, layered materials have attracted much attention. ${ }^{1}$ In particular, layered double hydroxides (LDHs) have been investigated for their potential application as electroactive materials due to their high surface area, chemical versatility, electrochemical stability as well as their inexpensive and abundant constituting elements. ${ }^{2,3}$

The structure of LDHs consists of stacks of positively charged metal hydroxides layers with charge balancing anions situated between the layers. They exhibit a typical formula $\left[\mathrm{M}^{\mathrm{II}}{ }_{1-\chi} \mathrm{M}^{\mathrm{III}} \chi(\mathrm{OH})_{2}\right]^{\chi+}\left(\mathrm{A}^{\mathrm{n}-}\right)_{\chi / \mathrm{n}} \cdot \mathrm{yH}_{2} \mathrm{O}$, where $\mathrm{M}^{\mathrm{II}}$ and $\mathrm{M}^{\mathrm{III}}$ are divalent and trivalent metals, respectively, such as $\mathrm{M}^{\mathrm{II}}=\mathrm{Mg}^{2+}, \mathrm{Zn}^{2+}, \mathrm{Ni}^{2+}, \mathrm{Co}^{2+}, \mathrm{Fe}^{2+}$ and $\mathrm{M}^{\mathrm{III}}=\mathrm{Al}^{3+}, \mathrm{Fe}^{3+}, \mathrm{Co}^{3+}$, $\mathrm{Ni}^{3+}, \mathrm{Cr}^{3+}$. The nature and charge of the components can be varied within a given compositional range (typically $0.20 \leq \mathrm{x} \leq 0.33)$ in addition, the interlayer anion $\left(\mathrm{A}^{\mathrm{n}-}\right)$ can be modified by anion exchange reactions in a broad range using both organic or inorganic molecules. 4 This wide chemical tunability allows the synthesis of multifunction- al LDHs with a great variety of properties that lead to their application in different fields such as energy storage, catalysis, anion exchange or magnetism, among others..$^{-8}$ In this framework, LDHs containing transition metals like $\mathrm{Ni}, \mathrm{Co}$, and $\mathrm{Fe}$ have shown excellent oxygen evolution reaction (OER) activity, being the most active composition at a ratio of $\mathrm{Ni}$ :Fe between $2: 1$ and 3:1.9,10 In the attempt to improve the electrochemical performance of LDHs-based electrodes, it has been reported successful tuning of the microstructure, the metal composition, the crystallinity and the particle size, which can provide more electroactive sites, leading to better performances., ${ }^{9,1,12}$ Beyond these well-studied parameters, interlayer anion modification has also been shown to be an effective approach for boosting the electrocatalytic activity of LDHs. Indeed, Hunter et al. synthesized nanometric $(<25 \mathrm{~nm})$ $\mathrm{NiFe-LDHs}$ by pulsed laser ablation in liquids, changing the identity of the intercalated gallery anions to influence the OER catalytic activity by local modification of $\mathrm{pK}_{\mathrm{a}}{ }^{13}$ However, only a reduced basal space region was studied (ca. $7.5-8.3 \AA$ ), showing that carbonate rapidly replaces the interlayer anion under environmental conditions. In this line, the intercalation of a large surfactant molecule like dodecyl sulphate has recently been reported as one effective approach to minimize the carbonate substitution 


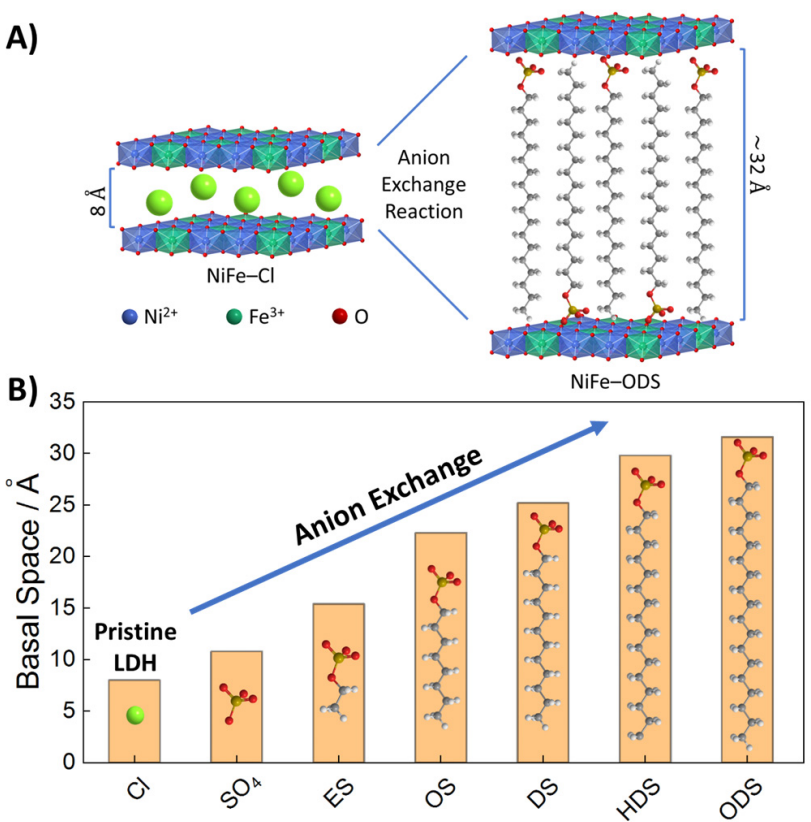

Figure 1. (A) Main scheme of the anion exchange reaction of an LDH with the corresponding increase of the basal space. (B) Basal space of the different intercalated LDHs according to the interlayer anion.

and to enhance the electrocatalytic activity of these layered materials. ${ }^{14}$ Unfortunately, so far the synthetic- and electrode preparation conditions reported in the literature vary substantially and there is a lack in the structural characterisation of these materials. This precludes a detailed understanding of intrinsic catalytic activity principles. For example, the role of crystallinity, chemical composition, particle size, morphology, and electrode preparations remain largely unexplored, all this precluding a deep understanding of the factors governing the OER activity. Along this front, a systematic study controlling all these parameters, deciphering the influence of interlayer space and determining the most effective materials is highly desirable.

On the other hand, it has been reported that the exfoliation of LDHs into $2 \mathrm{D}$ nanosheets result in vast improvements to overall OER performance correlated to the larger electrochemical area and the number of active sites. ${ }^{12,15}$ Taking into advantage the chemical flexibility of these layered materials, it is possible to envisage a precise molecular engineering leading to an electrochemical performance comparable to that of exfoliated nanosheets.

Keeping this in mind, we have attempted the synthesis by anion exchange reaction of a family of highly crystalline $\mathrm{NiFe-LDHs} \mathrm{endowed} \mathrm{with} \mathrm{similar} \mathrm{metal} \mathrm{composition,}$ morphology and dimensions, and modified with different interlayer anions (sulphate, ethyl sulphate, octyl sulphate, dodecyl sulphate, hexadecyl sulphate and octadecyl sulphate) in order to reach extreme ranges of basal spacing (from 8 to $32 \AA$, corresponding to the pristine chloride $\mathrm{LDH}$ and the octadecyl sulphate-intercalated $\mathrm{LDH}$, respectively, Figure 1). To achieve that, a non-aqueous methodology using triethanolamine as chelating reagent ${ }^{16,17}$ was followed to avoid the presence of $\mathrm{CO}_{3}{ }^{2-}$ in the reaction media, thus facilitating the anion exchange reaction. ${ }^{14}$ Notice that while $\mathrm{Al}^{3+}$-containing LDHs exhibit good results towards anion exchange replacements, ${ }^{18}$ this procedure is not so trivial for NiFe-LDHs. ${ }^{16,19,20}$ Then, once this anion exchange has been accomplished, a systematic study of the OER was possible to be carried out to precisely investigate the interlayer distance influence on the electrochemical properties.

Our results show that the interlayer increase leads to an excellent OER performance, with the optimal behaviour obtained for the NiFe-dodecyl sulphate. Another important finding is that the maximum increase in the interlayer distance (NiFe-octadecyl sulphate) allows for kinetic values comparable to benchmark NiFe-exfoliated nanosheets and with superior long-time stability, which will facilitate the future development of new highly efficient and robust hybrid electrocatalysts.

\section{EXPERIMENTAL}

\section{Chemicals}

$\mathrm{NiCl}_{2} \cdot 6 \mathrm{H}_{2} \mathrm{O}, \quad \mathrm{FeCl}_{3} \cdot 6 \mathrm{H}_{2} \mathrm{O}$, triethanolamine (TEA, $\left.\mathrm{C}_{6} \mathrm{H}_{15} \mathrm{NO}_{3}\right)$, sodium sulphate $\left(\mathrm{NaO}_{4} \mathrm{~S}\right)$ sodium octyl sulphate $\left(\mathrm{C}_{8} \mathrm{H}_{17} \mathrm{NaO}_{4} \mathrm{~S}\right)$, sodium dodecyl sulphate $\left(\mathrm{C}_{12} \mathrm{H}_{17} \mathrm{NaO}_{4} \mathrm{~S}\right)$, sodium hexadecyl sulphate $\left(\mathrm{C}_{16} \mathrm{H}_{35} \mathrm{NaO}_{4} \mathrm{~S}\right)$, sodium octadecyl sulphate $\left(\mathrm{C}_{18} \mathrm{H}_{37} \mathrm{NaO}_{4} \mathrm{~S}\right)$ and polytetrafluoroethylene (PTFE) were purchased from SigmaAldrich. Sodium ethyl sulphate $\left(\mathrm{C}_{2} \mathrm{H}_{5} \mathrm{NaO}_{4} \mathrm{~S}\right)$ was purchased from TCI. Potassium hydroxide $\mathrm{KOH}$ (99.99\%) was purchased from Panreac. All chemicals were used as received. Ethanol absolute $(\mathrm{EtOH})$ was purchased from Panreac. Carbon black, acetylene 50\% compressed, was obtained from Alfa Aesar (99.9\%). Milli-Q water was obtained from a Millipore Milli-Q equipment.

\section{Synthesis of LDHs}

Synthesis of $\mathrm{NiFe}-\mathrm{Cl} \mathrm{LDH}$. NiFe-Cl LDH with $\mathrm{Ni} / \mathrm{Fe}$ ratio 3:1 was synthesized using a hydrothermal method adapted from Dang et al. ${ }^{14}$ In a typical procedure, $0.45 \mathrm{mmol}$ of $\mathrm{FeCl}_{3} \cdot 6 \mathrm{H}_{2} \mathrm{O}$ were added to a centrifuge tube containing $900 \mu \mathrm{mol}$ of TEA. Afterwards, a solution with $1.35 \mathrm{mmol}$ of $\mathrm{NiCl}_{2} \cdot 6 \mathrm{H}_{2} \mathrm{O}$ and $1.2 \mathrm{~g}$ of TEA $(8 \mathrm{mmol})$ dissolved in 90 $\mathrm{mL}$ of a mixture 1:1 (v:v) ethanol:water was added. The resulting solution was mixed thoroughly until complete dissolution and transferred to a Teflon-lined stainlesssteel autoclave. The autoclave was placed in a preheated oven at $120^{\circ} \mathrm{C}$ for $6 \mathrm{~h}$. Finally, it was cooled down to room temperature, and the final brown powder was filtered and washed with $\mathrm{H}_{2} \mathrm{O}$ and $\mathrm{EtOH}$ several times and dried during $24 \mathrm{~h}$ in vacuum.

Synthesis of $\mathrm{NiFe}^{-\mathrm{SO}_{4}} \mathrm{LDH}$. 0.25 M of sodium sulphate was dissolved in $50 \mathrm{~mL}$ of a $1: 1(\mathrm{v}: \mathrm{v})$ ethanol:water mixture with magnetic stirring at $65{ }^{\circ} \mathrm{C}$ and under argon atmosphere. After 30 minutes, $50 \mathrm{mg}$ of $\mathrm{LDH} \mathrm{NiFe}-\mathrm{Cl}$ were 
added. The temperature was set at $50{ }^{\circ} \mathrm{C}$ and the reaction was kept for $48 \mathrm{~h}$. Finally, the mixture was filtered, washed with Milli-Q water and EtOH, and dried in vacuum.

Synthesis of NiFe-ES LDH. $7.5 \mathrm{mM}$ of sodium ethyl sulphate was dissolved in $50 \mathrm{~mL}$ of a $1: 1$ (v:v) ethanol:water mixture. Then, $50 \mathrm{mg}$ of $\mathrm{NiFe}-\mathrm{Cl} \mathrm{LDH}$ were added, and the reaction was maintained for $48 \mathrm{~h}$ at room temperature under Ar atmosphere and magnetic stirring. Finally, the final mixture was filtered, and the yellow powder was washed with $\mathrm{H}_{2} \mathrm{O}$ and $\mathrm{EtOH}$ several times and dried during $24 \mathrm{~h}$ in vacuum.

Synthesis of NiFe-OS LDH. $7.5 \mathrm{mM}$ of sodium octyl sulphate was dissolved in $50 \mathrm{~mL}$ of a $1: 1 \quad(\mathrm{v}: \mathrm{v})$ ethanol:water mixture. Then, $30 \mathrm{mg}$ of $\mathrm{NiFe}-\mathrm{Cl} \mathrm{LDH}$ were added, and the reaction was maintained for $48 \mathrm{~h}$ at room temperature under $\mathrm{Ar}$ atmosphere and magnetic stirring. Finally, the final mixture was filtered, and the yellow powder was washed with $\mathrm{H}_{2} \mathrm{O}$ and $\mathrm{EtOH}$ several times and dried during $24 \mathrm{~h}$ in vacuum.

Synthesis of NiFe-DS LDH. 0.2 M of sodium dodecyl sulphate was dissolved in $50 \mathrm{~mL}$ of a $1: 1 \quad(\mathrm{v}: \mathrm{v})$ ethanol:water mixture. Then, $200 \mathrm{mg}$ of $\mathrm{NiFe}-\mathrm{Cl} \mathrm{LDH}$ were added, and the reaction was maintained for $12 \mathrm{~h}$ at room temperature under $\mathrm{Ar}$ atmosphere and magnetic stirring. Finally, the final mixture was filtered, and the yellow powder was washed with $\mathrm{H}_{2} \mathrm{O}$ and EtOH several times and dried during $24 \mathrm{~h}$ in vacuum.

Synthesis of NiFe-HDS LDH. $2.5 \mathrm{mM}$ of sodium hexadecyl sulphate was dissolved in $50 \mathrm{~mL}$ of a $1: 1 \quad(\mathrm{v}: \mathrm{v})$ ethanol:water mixture. Then, $50 \mathrm{mg}$ of $\mathrm{NiFe}-\mathrm{Cl} \mathrm{LDH}$ were added, and the reaction was maintained for $12 \mathrm{~h}$ at room temperature under Ar atmosphere and magnetic stirring. Finally, the final mixture was filtered, and the yellow powder was washed with $\mathrm{H}_{2} \mathrm{O}$ and $\mathrm{EtOH}$ several times and dried during $24 \mathrm{~h}$ in vacuum.

Synthesis of NiFe-ODS LDH. o.o1 M of sodium octadecyl sulphate was dissolved in $50 \mathrm{~mL}$ of a $1: 1$ (v:v) ethanol:water mixture. Then, $200 \mathrm{mg}$ of $\mathrm{NiFe}-\mathrm{Cl} \mathrm{LDH}$ were added, and the reaction was maintained for $12 \mathrm{~h}$ at room temperature under $\mathrm{Ar}$ atmosphere and magnetic stirring. Finally, the final mixture was filtered, and the yellow powder was washed with $\mathrm{H}_{2} \mathrm{O}$ and EtOH several times and dried during $24 \mathrm{~h}$ in vacuum.

\section{Structural characterization}

X-ray powder diffraction (XRPD) patterns were obtained with a Bruker D8 Advance A25 using the copper radiation $\left(\mathrm{Cu}-\mathrm{K}_{\alpha}=1.54178 \AA\right)$ in the $2-70^{\circ}$ region. Attenuated total reflectance Fourier-transform infrared (ATR-FTIR) were collected in an Agilent Cary 630 FTIR spectrometer in the 4000-650 $\mathrm{cm}^{-1}$ range in absence of $\mathrm{KBr}$ pellets. Dynamic Light Scattering (DLS) measurements: DLS measurements were performed at room temperature with a Zetasizer Nano ZS instrument (Malvern Instruments Ltd.). Atomic Force Microscopy (AFM) measurements were collected in a Multimode atomic force microscope from Veeco, using tapping mode in air at room temperature. Processing and analysis of the images were carried out using the Nanotec WSxM 6.o software. ${ }^{21}$ Field emission scanning electron microscopy (FESEM) and energydispersive X-ray (EDX) spectroscopy mappings studies were carried out on a Hitachi S-480o microscope at an accelerating voltage of $20 \mathrm{kV}$ and $30 \mathrm{~s}$ of $\mathrm{Au} / \mathrm{Pd}$ metallization of the samples. Transmission electron microscopy (TEM): TEM studies were carried out on a JEM-1010 operating at $8 \mathrm{o} \mathrm{kV}$. Samples were prepared by dropping suspensions on lacey formvar/carbon copper grids (300 mesh). Thermogravimetric analysis (TGA) of all samples was carried out in a Mettler Toledo TGA/DSC 2 apparatus in the $30-800{ }^{\circ} \mathrm{C}$ temperature range at $10{ }^{\circ} \mathrm{C} \cdot \mathrm{min}^{-1}$ scan rate and an air flow of $100 \mathrm{~mL} \cdot \mathrm{min}^{-1}$. Carbon, nitrogen, hydrogen, and sulphur contents were determined by microanalytical procedures by using a LECO CHNS-932. X-ray photoelectron spectroscopy (XPS) measurements were performed in an ultrahigh vacuum system ESCALAB210 (base pressure $1.0 \times 10^{-10} \mathrm{mbar}$ ) from Thermo VG Scientific. Photoelectrons were excited by using the $\mathrm{Mg}-\mathrm{K} \alpha$ line $(1253.6 \mathrm{eV})$. All spectra were referred to the Fermi level. Nitrogen adsorption/desorption isotherms at $77 \mathrm{~K}$ and carbon dioxide adsorption isotherms at $298 \mathrm{~K}$ were collected in an AUTOSORB-6 apparatus. Samples were previously degassed at $423 \mathrm{~K}$ for $12 \mathrm{~h}$ under vacuum with an AUTOSORB DEGASSER. Magnetic data were collected over the bulk material with a Quantum Design superconducting quantum interference device (SQUID) MPMS-XL-5. The magnetic susceptibility of the samples was corrected from the diamagnetic contributions of their atomic constituents as deduced from Pascal's constant tables and the sample holder. The DC data were obtained under an external applied field of 100 or 1000 Oe in the 2$300 \mathrm{~K}$ temperature range.

\section{Electrode preparation}

For the electrochemical measurements, a mixture of the $\mathrm{LDH}$, acetylene black, and PTFE in ethanol in a mass ratio of 80:10:10 was prepared and deposited on a nickel foam electrode. The as-prepared electrode was let dried for $2 \mathrm{~h}$ at $80{ }^{\circ} \mathrm{C}$. Each working electrode contained about $0.4 \mathrm{mg}$ of electroactive material (i.e. the metal hydroxides) and had a geometric surface area of about $2 \mathrm{~cm}^{2}$. Thus, the electrode mass loading achieved was $0.2 \mathrm{mg} \cdot \mathrm{cm}^{-2}$. A typical three-electrode cell equipped with a steel sheet as the counter electrode and a Metrohm $\mathrm{Ag} / \mathrm{AgCl}(3 \mathrm{M} \mathrm{KCl})$ as the reference electrode was used for the electrochemical characterization of the nanocomposite material trapped by the working electrodes. Also, additional measurements were performed using glassy carbon rotating disc electrode (RDE) following similar procedure reported by Dang et al. ${ }^{14}$ Electrodes were prepared by adding $25 \mu \mathrm{L}$ of $5 \%$ Nafion solution to $5 \mathrm{mg}$ of $\mathrm{LDH}$ powder and $2.5 \mathrm{mg}$ of graphitized carbon and then dispersed in $1.25 \mathrm{~mL}$ of $1: 1$ (v:v) $\mathrm{EtOH} / \mathrm{H}_{2} \mathrm{O}$ and sonicated for 20 min. $3 \mu \mathrm{L}$ of this dispersion was drop-casted onto a previously polished 
(sequentially with 1.0, 0.3 and $0.05 \mu \mathrm{m}$ alumina powder) 3 $\mathrm{mm}$ diameter RDE and dried at room temperature for 30 $\min$.

\section{Electrochemical characterization}

Prior to OER experiments, cyclic voltammetry curves $(\mathrm{CVs})$ were performed between $0.9-1.7 \mathrm{~V}$ vs RHE at different scan rates $\left(100,50,20,10\right.$ and $\left.5 \mathrm{mV} \cdot \mathrm{s}^{-1}\right)$. Linear sweep voltammetry (LSV) measurements were carried out at $5 \mathrm{mV} \cdot \mathrm{s}^{-1}$ in a previously $\mathrm{N}_{2}$ purged $1 \mathrm{M} \mathrm{KOH}$ aqueous solution. The measurements were performed at least three times for every sample using different electrodes. All electrochemical experiments were done without $i R$ correction, in order to make a fair comparison of the intrinsic electrocatalytic behaviour of each sample instead of searching the best performance of the electrode. OER tests were performed at room temperature using a Gamry 1010 E potentiostat-galvanostat controlled by Gamry software. RDE was used rotating at $1200 \mathrm{rpm}$ for NiFe-ODS and an exfoliated NiFe to determine their electrocatalytic performance.

The turnover frequency (TOF) values were calculated from the equation:

$$
\mathrm{TOF}=\frac{\mathrm{jA}}{4 \mathrm{Fm}}
$$

where $j$ is the current density at a given overpotential of $0.3 \mathrm{~V}, \mathrm{~A}$ is the surface area of the working electrode, $\mathrm{F}$ is the Faraday constant and $\mathrm{m}$ is the number of moles of metal loaded on the electrode (i.e. $\mathrm{Ni}$ and $\mathrm{Fe}$ ).

Electrochemical Surface Area (ECSA) was acquired by measuring the current associated with double-layer capacitance from the scan-rate dependence of CVs. The ECSA was measured on the working electrodes after LSV measurements. The potential range used for the CVs was $0.2-0.3 \mathrm{~V}$ versus $\mathrm{Ag} / \mathrm{AgCl}(3 \mathrm{M} \mathrm{KCl})$. The scan rates were 5, 10, 20, 50 and $100 \mathrm{mV} \mathrm{s}^{-1}$. The double-layer capacitance was calculated by plotting the $\mathrm{j}_{\mathrm{a}}-\mathrm{j}_{\mathrm{c}} / 2$ (anodic versus cathodic currents) at $0.25 \mathrm{~V}$ versus $\mathrm{Ag} / \mathrm{AgCl}(3 \mathrm{M} \mathrm{KCl})$ against the scan rate. ECSA measurements were obtained in an Autolab PGSTAT 128N potentiostat/galvanostat.

Electrochemical impedance spectroscopy (EIS) measurements were carried out by applying an AC amplitude of 10 $\mathrm{mV}$ in the frequency range of $0.01-10^{4} \mathrm{~Hz}$ at an overpotential of $0.3 \mathrm{~V}$ using a Gamry 1010E potentiostat-galvanostat. Measurements were recorded after an equilibration time of $30 \mathrm{~s}$ upon an overpotential of $0.3 \mathrm{~V}$. EIS data were analysed and fitted by means of Gamry Echem Analyst v. 7.07 software.

Diffusion coefficients were calculated taking into account that the oxidation of nickel is diffusion limited. Then, according to the Randles-Sevcik equation:

$$
\mathrm{i}_{\mathrm{p}}=2.69 \cdot 10^{-5} \mathrm{n}^{3 / 2} \mathrm{~A} \sqrt{\mathrm{D}_{\mathrm{o}}} \sqrt{\mathrm{v}} \sqrt{\mathrm{C}_{\mathrm{o}}}
$$

where $i_{p}$ is the current peak in $A ; n$ is the number of electrons transferred (usually 1 ); $A$ is the surface area of the electrode, $2 \mathrm{~cm}^{2} ; \mathrm{D}_{\mathrm{o}}$ is the diffusion coefficient of the ratelimiting species in $\mathrm{cm}^{2} \cdot \mathrm{s}^{-1} ; \mathrm{v}$ is the scan rate in $\mathrm{V} \cdot \mathrm{s}^{-1} ; \mathrm{C}_{\mathrm{o}}$ is the proton concentration in the solution in $\mathrm{mol} \cdot \mathrm{cm}^{-3}$. To estimate the proton concentration, it is assumed to be the same as $\mathrm{Ni}(\mathrm{OH})_{2}$ following the stoichiometry of the reaction: $\mathrm{Ni}(\mathrm{OH})_{2} \rightarrow \mathrm{NiOOH}+\mathrm{H}^{+}+\mathrm{e}^{-}$. The electrodes were prepared with the same electroactive metal mass loading. Thus, the concentration of $\mathrm{Ni}(\mathrm{OH})_{2}$ in all cases is the same.

Stability tests were carried out at constant current densities of 5 and $10 \mathrm{~mA} \cdot \mathrm{cm}^{-2}$ and at constant overpotentials of $300 \mathrm{mV}$ and $400 \mathrm{mV}$ for $2 \mathrm{~h}$ for $\mathrm{NiFe}-\mathrm{Cl}$ and $\mathrm{NiFe}-\mathrm{ODS}$. In addition, the stability of NiFe-ODS and an exfoliated $\mathrm{NiFe}-\mathrm{LDH}$ was performed under a constant current density of $10 \mathrm{~mA} \cdot \mathrm{cm}^{-2}$ during 48 hours.

ATR-FTIR spectra after the different electrochemical tests were recorded by extracting the $\mathrm{LDH}$ material from the $\mathrm{Ni}$ foam using a sonication procedure followed by a washing step.

\section{RESULTS AND DISCUSSION}

Pristine $\mathrm{NiFe}-\mathrm{Cl} \mathrm{LDH}$ was synthesized using triethanolamine as chelating reagent ${ }^{16,17,20}$ and following a modified methodology in absence of urea described by Dang et al. ${ }^{14}$ By subsequent anion exchange of the chloride form with five different $n$-alkyl-sulphate derivatives, we obtained a family of materials with an increasing number of carbon
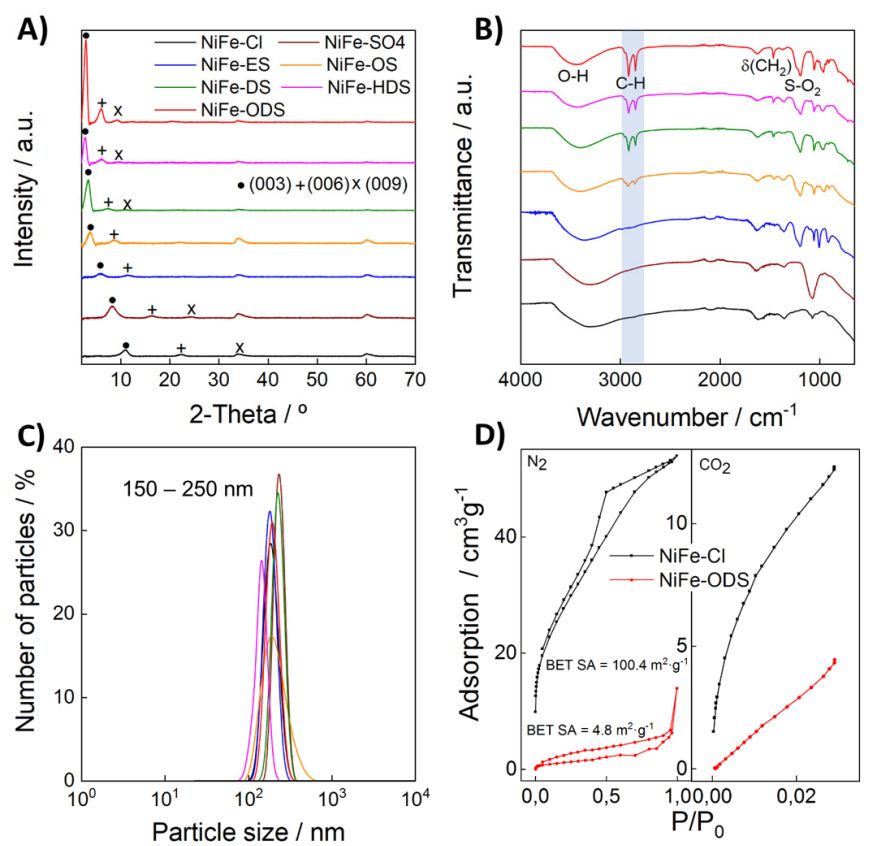

Figure 2. Physical characterization of the NiFe-LDH family. (A) XRPD diffractograms highlighting the main basal reflections. (B) IR spectra (C) DLS measurements denoting the average size of the particles. (D) $\mathrm{N}_{2}$ adsorption-desorption and $\mathrm{CO}_{2}$ adsorption isotherms for $\mathrm{NiFe}-\mathrm{Cl}$ and $\mathrm{NiFe}-\mathrm{ODS}$ samples. 
atoms: 2 for the ethyl (-ES), 8 for the octyl (-OS), 12 for the dodecyl (-DS), 16 for the hexadecyl (-HDS) and 18 for the octadecyl (-ODS) form. ${ }^{18}$ It is worth to remark that every surfactant-intercalated material requires some specific synthetic conditions. LDH phases were confirmed via XRPD pattern (Figure $2 \mathrm{~A}$ and $\mathrm{S}_{1}$ ), highlighting the main basal reflections that can be found in hydrotalcite-like materials (JCPDS 22-700). ${ }^{22,23}$ The main (003), (oo6) and (oo9) peaks are related to the basal space of the LDH, therefore dependent on the size of the interlayer anion. We observe that the peaks shift towards lower $2-\theta$ values and increase their intensity as long as the length of the interlamellar anion rises. ${ }^{18}$ For the pristine $\mathrm{NiFe}-\mathrm{Cl}$ material, we observe a basal space which is $8.0 \AA$, in good agreement with that found in the literature. ${ }^{14}$ For the longest interlayer distance (NiFe-ODS) the basal space increases up to $31.6 \AA$, therefore confirming the successful anion exchange reaction and, to the best of our knowledge, the largest basal space reported so far for a NiFe-LDH. ${ }^{18}$ No contaminant phases were observed, confirming that the anion exchange reactions do not affect the overall crystallinity of the layered hosts. In addi-
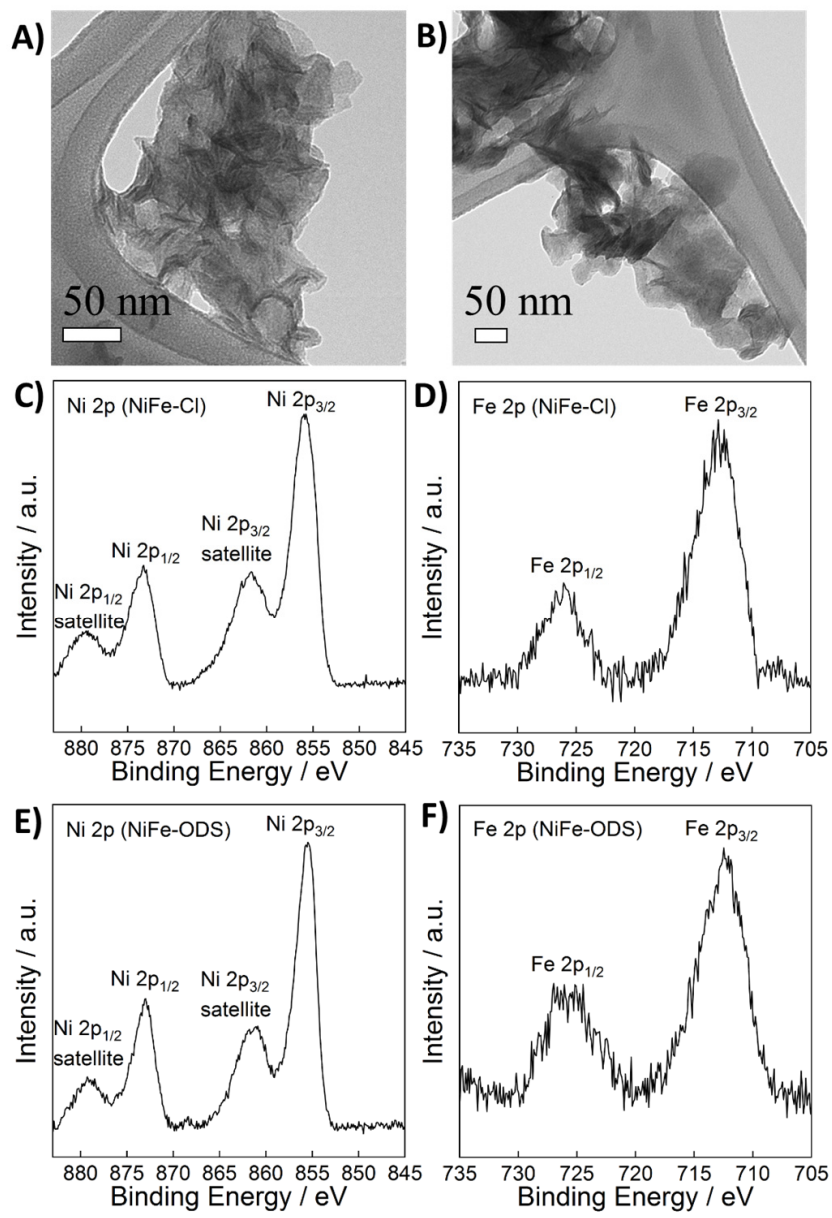

Figure 3. Electron microscopy characterization. TEM images of (A) NiFe-Cl and (B) NiFe-ODS. XPS spectra of (C, D) NiFe$\mathrm{Cl}$ and $(\mathrm{E}, \mathrm{F}) \mathrm{NiFe}-\mathrm{ODS}$. tion, the peak observed near $60^{\circ}$ is indicative of the structural ordering of the material. ${ }^{22}$ The absence of extrinsic magnetic impurities such as Fe-spinels, which can be electrocatalytic active, has been further demonstrated by temperature-dependent magnetic susceptibility measurements, $\chi$, reported as $\chi \mathrm{T}$ versus $\mathrm{T}$ showing the absence of a temperature-independent component (Figure S2). ${ }^{17}$

Attenuated total reflectance Fourier-transform infrared (ATR-FTIR) spectroscopy was carried out in order to confirm the nature of the interlayer anion (Figure $2 \mathrm{~B}$ ), highlighting the $\mathrm{C}-\mathrm{H}$ stretching bands at 2917 and 2845 $\mathrm{cm}^{-1}$ plus the sulphate bands at $\mathrm{ca}$. 1190 and $1050 \mathrm{~cm}^{-1}$ in the surfactant-intercalated samples. ${ }^{18,24}$ While the $v\left(\mathrm{SO}_{2}\right)$ band in the surfactant-intercalated LDHs is found at ca. $1219 \mathrm{~cm}^{-1}$, for the $\mathrm{NiFe}-\mathrm{SO}_{4}$ sample it is observed at $c a$. $1100 \mathrm{~cm}^{-1}$. This shift is in good agreement with that observed in the literature due to the loss of interaction between the headgroup and the carboxylic chain. ${ }^{24,25}$ In order to further corroborate this effect, we measured the IR spectra of $\mathrm{NaSO}_{4}$ and sodium dodecyl sulphate (Figure $\mathrm{S}_{3}$ ). According to the work of Lagaly et al., we can expect a bilayer arrangement of the surfactant in the interlamellar space exhibiting tilt angles in the $9-24^{\circ}$ region. ${ }^{26}$

TGA/DSC analysis can be found in Figure $\mathrm{S}_{4}$, depicting the typical profile for an LDH material, pointing out an initial weight loss in the $25-220{ }^{\circ} \mathrm{C}$ range related to the removal of physisorbed $\mathrm{H}_{2} \mathrm{O}$ and solvation molecules, and a second weight loss in the $220-600{ }^{\circ} \mathrm{C}$ range, ascribed with the decomposition of the interlayer anion as well as the dehydroxylation of the LDH layers. ${ }^{22,27-29}$ In addition, dynamic light scattering (DLS) measurements were carried out in order to evaluate the average size of the $\mathrm{LDH}$ particles (Figure ${ }_{2} \mathrm{C}$ and $\mathrm{S}_{5}$ ). We found mean sizes in the range of $150-250 \mathrm{~nm}$ for all samples, which agrees well with other nanometric LDHs synthesised using nonaqueous co-precipitation routes. ${ }^{10,27,30,31}$ The lateral size and thickness of NiFe-ODS was further confirmed by atomic force microscopy (AFM) (Figure S6). Moreover, to further investigate the anion exchange process, a complete porosity analysis was carried out for the $\mathrm{NiFe}-\mathrm{Cl}$ and $\mathrm{NiFe-ODS}$ samples, endowed with the lowest and highest interlayer spaces, respectively (Figure $2 \mathrm{D}$ and Table $\mathrm{S} 1$ ). The $\mathrm{N}_{2}$ isotherms at $77 \mathrm{~K}$ exhibited a Type IV behaviour, in good agreement with previous reports. ${ }^{22,32,33}$ While the pristine $\mathrm{NiFe}-\mathrm{Cl}$ displays typical values of BrunauerEmmett-Teller (BET) surface area of $100 \mathrm{~m}^{2} \cdot \mathrm{g}^{-1}$, the surface area abruptly decreases below $10 \mathrm{~m}^{2} \cdot \mathrm{g}^{-1}$ after the anion exchange reactions. This shift to lower values is reported in the literature for other hybrid LDHs intercalated with large organic molecules ${ }^{34-36}$. The effect is caused by the densely packed intercalated surfactant molecules, which block the passage of $\mathrm{N}_{2}$ molecules during the adsorption procedure. ${ }^{37,38}$ Additionally, we measured the $\mathrm{CO}_{2}$ adsorption at $298 \mathrm{~K}$ in order to evaluate the presence of narrow micropores of $<0.7 \mathrm{~nm}$. In this context, we

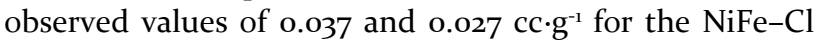
and $\mathrm{NiFe-ODS}$ samples, respectively, hence exhibiting the NiFe-ODS a narrow micropore contribution of $c a .73$ 
Table 1. Compositional information of the NiFe-LDH family

\begin{tabular}{|c|c|c|c|c|}
\hline Sample & $\begin{array}{c}\text { Carbon } \\
\text { Number }\end{array}$ & $\begin{array}{c}\text { Basal } \\
\text { Space }(\AA)\end{array}$ & Molecular Formula & Ratio Ni:Fe \\
\hline $\mathrm{NiFe}-\mathrm{Cl}$ & 0 & 8.0 & {$\left[\mathrm{Ni}_{0.73} \mathrm{Fe}_{0.27}(\mathrm{OH})_{2}\right]^{0.27+}\left(\mathrm{Cl}^{-}\right)_{0.27} \cdot 0.44 \mathrm{H}_{2} \mathrm{O} \cdot 0.16 \mathrm{C}_{2} \mathrm{H}_{5} \mathrm{OH}$} & 2.73 \\
\hline $\mathrm{NiFe}-\mathrm{SO}_{4}$ & 0 & 10.8 & {$\left[\mathrm{Ni}_{0.73} \mathrm{Fe}_{0.27}(\mathrm{OH})_{2}\right]^{0.27+}\left(\mathrm{SO}_{4}{ }^{2-}\right)_{0.135} \cdot 0.25 \mathrm{H}_{2} \mathrm{O} \cdot 0.26 \mathrm{C}_{2} \mathrm{H}_{5} \mathrm{OH}$} & 2.75 \\
\hline $\mathrm{NiFe}-\mathrm{ES}$ & 2 & 15.4 & {$\left[\mathrm{Ni}_{0.73} \mathrm{Fe}_{0.27}(\mathrm{OH})_{2}\right]^{0.27+}\left(\mathrm{ES}^{-}\right)_{0.17}\left(\mathrm{Cl}^{-}\right)_{0.10} \cdot 0.39 \mathrm{H}_{2} \mathrm{O} \cdot 0.20 \mathrm{C}_{2} \mathrm{H}_{5} \mathrm{OH}$} & 2.68 \\
\hline $\mathrm{NiFe}-\mathrm{OS}$ & 8 & 22.3 & {$\left[\mathrm{Ni}_{0.74} \mathrm{Fe}_{0.26}(\mathrm{OH})_{2}\right]^{0.26+}\left(\mathrm{OS}^{-}\right)_{0.20}\left(\mathrm{Cl}^{-}\right)_{0.06} \cdot 0.60 \mathrm{H}_{2} \mathrm{O}$} & 2.85 \\
\hline $\mathrm{NiFe}-\mathrm{DS}$ & 12 & 25.2 & {$\left[\mathrm{Ni}_{0.73} \mathrm{Fe}_{0.27}(\mathrm{OH})_{2}\right]^{0.27+}\left(\mathrm{DS}^{-}\right)_{0.22}\left(\mathrm{Cl}^{-}\right)_{0.05} \cdot 0.60 \mathrm{H}_{2} \mathrm{O}$} & 2.74 \\
\hline $\mathrm{NiFe}-\mathrm{HDS}$ & 16 & 29.8 & {$\left[\mathrm{Ni}_{0.74} \mathrm{Fe}_{0.26}(\mathrm{OH})_{2}\right]^{0.26+}\left(\mathrm{HDS}^{-}\right)_{0.21}\left(\mathrm{Cl}^{-}\right)_{0.05} \cdot 0.5 \mathrm{H}_{2} \mathrm{O}$} & 2.81 \\
\hline $\mathrm{NiFe}-\mathrm{ODS}$ & 18 & 31.6 & {$\left[\mathrm{Ni}_{0.74} \mathrm{Fe}_{0.26}(\mathrm{OH})_{2}\right]^{0.26+}\left(\mathrm{ODS}^{-}\right)_{0.22}\left(\mathrm{Cl}^{-}\right)_{0.04} \cdot 0.8 \mathrm{H}_{2} \mathrm{O} \cdot 0.2 \mathrm{C}_{2} \mathrm{H}_{5} \mathrm{OH}$} & 2.86 \\
\hline
\end{tabular}

Basal space was calculated as $\left(\mathrm{d}_{\mathrm{oo} 3}+2 \mathrm{~d}_{\mathrm{oo6}}+3 \mathrm{~d}_{\mathrm{oog}}\right) / 3$

$\%$ of that calculated for the $\mathrm{NiFe}-\mathrm{Cl}$ material. Both samples exhibited larger micropore volume $(<0.7 \mathrm{~nm})$ when obtained from the $\mathrm{CO}_{2}$-adsorption isotherms than the total micropore volume extracted from the $\mathrm{N}_{2}$-adsorption isotherms. This is indicative of the presence of narrow constrictions which favour the insertion of $\mathrm{CO}_{2}$ molecules. 39 Additionally, the near to zero value of total micropore volume for $\mathrm{N}_{2}$ molecules observed in the NiFeODS sample indicates that all diffusion processes for hydroxyl groups came from the ultramicropore $(<0.7 \mathrm{~nm})$ regime. $4^{\circ}$ In order to check the stability of the samples after the thermal activation prior to the isotherm experiments ( $423 \mathrm{~K}$ for $12 \mathrm{~h}$ under vacuum), XRPD was measured on the activated samples confirming that the LDH phase remains unaltered after the activation process (Figure $\mathrm{S}_{7}$ ).

The morphology of the samples was unveiled by means of field-effect scanning electron microscopy (FESEM) and transmission electron microscopy (TEM, Figure 3A-B and Figure S8 and S9). In the light of the FESEM images, we observed that the LDH crystallites tend to assemble into bigger aggregates with average lateral sizes of $c a .1 .8 \mu \mathrm{m}$ (Figure S10). TEM pictures allowed us a better identification of the small particles composing the aggregates, in good agreement with the DLS measurement (ca. $150-250$ $\mathrm{nm}$ ). After the anion exchange reactions, the morphology was conserved, with no significant variation in the dimensions of the different intercalated samples. Whereas $\mathrm{Al}^{3+}$ containing LDHs synthesized in presence of ammonia releasing reagents (ARR) exhibited a typical well-defined hexagonal morphology ${ }^{41}$, the here reported NiFe-LDHs displayed smaller particles with less-defined morphology. This is due to the absence of urea, which allows to directly synthesize $\mathrm{NiFe}-\mathrm{Cl}$ avoiding the presence of $\mathrm{CO}_{3}{ }^{2-}$ in the reaction media. ${ }^{14}$ Energy-dispersive X-ray (EDX) spectroscopy mappings confirmed the homogeneity of the samples (Figure S11). Table 1 and $S_{1}$ shows the molecular formula of the LDHs calculated based on the TGA, EDX and elemental analysis results.

XPS analysis was carried out for the NiFe-LDH family in order to evidence the oxidation states of $\mathrm{Ni}$ and $\mathrm{Fe}$ (Figure ${ }_{3} \mathrm{C}-\mathrm{F}$ and $\mathrm{S}_{12}$ ). As reported in the literature for these materials, the presence of $\mathrm{Ni}$ (II) was confirmed according to its main peaks at $856\left(\mathrm{Ni} 2 \mathrm{p}_{3 / 2}\right)$ and $874\left(\mathrm{Ni} 2 \mathrm{p}_{1 / 2}\right) \mathrm{eV}$, combined with their satellite peaks found at 862 and 880 $\mathrm{eV}$, respectively. On the other hand, the presence of Fe (III) can be demonstrated by the characteristic peaks at 713 and $727 \mathrm{eV}$, related with the $\mathrm{Fe} 2 \mathrm{p}_{3 / 2} \mathrm{Fe} 2 \mathrm{p}_{1 / 2}$ signals, respectively. ${ }^{23,42-44}$

As described above, all samples were fully characterized and own very similar properties that strongly influence the electrocatalytic behaviour (morphology, composition, crystallinity, dimensions). Although these properties are not optimized for electrocatalysis, they must stay invariable with the purpose of determining, by a systematic study, the influence of the interlayer distance on the electrochemical performance.

The whole NiFe-LDH family was investigated as OER electrocatalysts using Ni foam collectors. Figure $\mathrm{S}_{13}$ displays the CVs of NiFe-LDHs performed at different scan rates. The presence of a redox peak between $1.3-1.5 \mathrm{~V}$ vs RHE is observed, which is related to the Ni redox processes. ${ }^{27,45}$ Electrocatalytic activity of the whole series was tested and compared by measuring LSV (Figure $4 \mathrm{~A}$ ) until 2.o V vs RHE. The electrocatalytic reaction follows different trends. In fact, the current greatly increases upon the augmentation of the basal space (Figure $\mathrm{S}_{14}$ ). This can be noticed in the inset figure where it is represented the overpotential required at different current densities. At low current densities, NiFe-DS, NiFe-HDS and NiFeODS are the LDHs that take lower overpotential values. Nonetheless, at current densities over $50 \mathrm{~mA} \cdot \mathrm{cm}^{-2}, \mathrm{NiFe}-$ HDS and NiFe-ODS present the best electrocatalytic response. It is worth to mention that for each sample, 5 CVs were recorded showing a similar registered signal indicating high resilience during the OER experiments (see Figure S14).

To properly analyse the electrocatalytic behaviour, four key parameters were compared: the Tafel slopes, the overpotential needed at $10 \mathrm{~mA} \cdot \mathrm{cm}^{-2}$, the current density measured at an overpotential of $0.3 \mathrm{~V}$ and the turnover frequency (TOF) values. All parameter values are reported in Table $\mathrm{S}_{3}$. Figures $4 \mathrm{~B}$ and $\mathrm{S}_{15}$ show the Tafel slope values calculated from Figure $4 \mathrm{~A}$. Interestingly, the Tafel slope values decrease from $101 \pm 2 \mathrm{mV} \cdot \mathrm{dec}^{-1}$ to $73 \pm 2$ $\mathrm{mV} \cdot \mathrm{dec}^{-1}$ when changing chloride by sulphates and their 

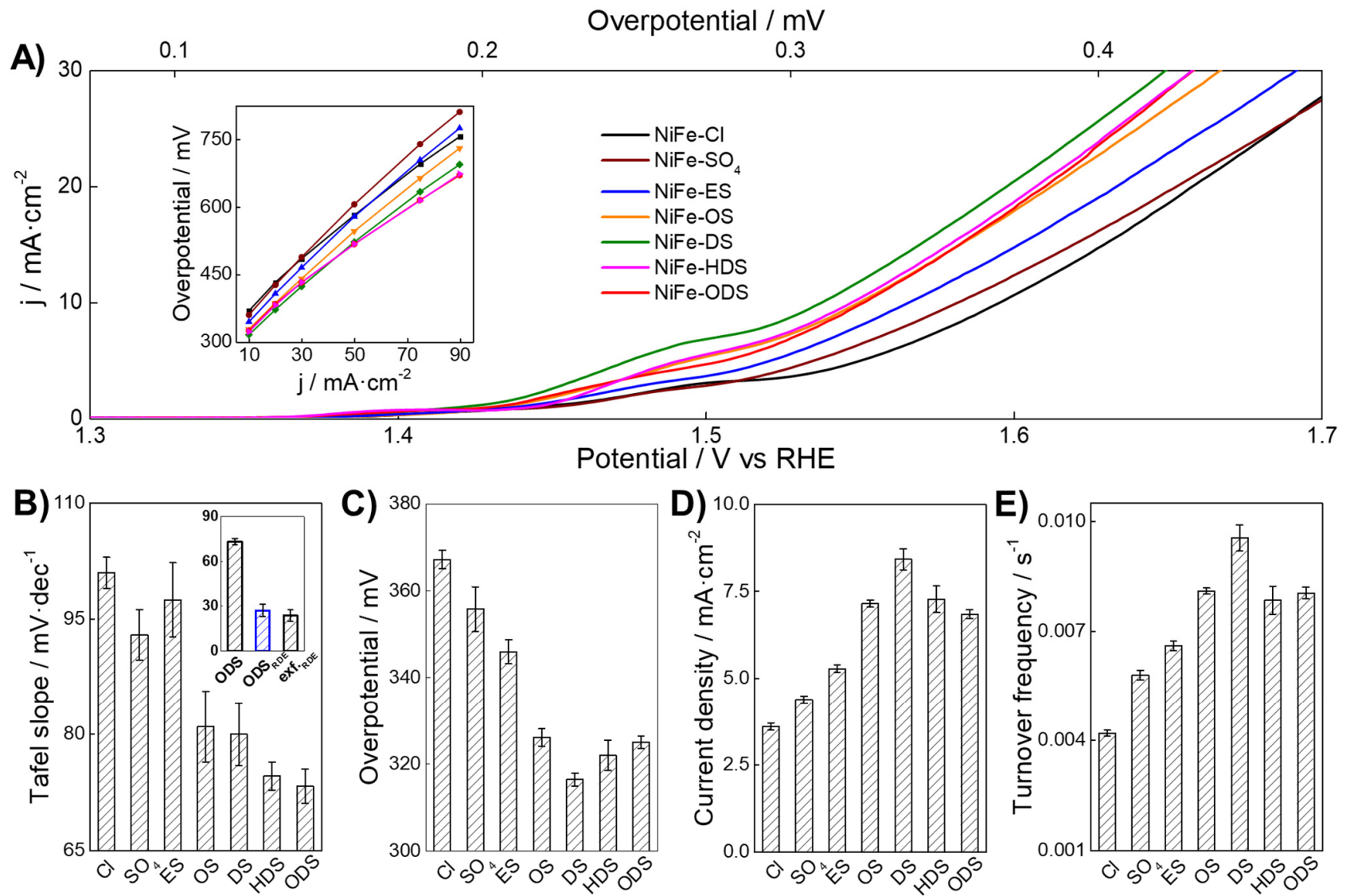

Figure 4. A) Lineal Sweep Voltammetry (LSV) of various NiFe-LDHs with different interlayer anions at $5 \mathrm{mV} \cdot \mathrm{s}^{-1}$ in $1 \mathrm{M} / \mathrm{KOH}$ solution. Inset: Overpotential measured at different current densities. B) Tafel slopes calculated from LSV data. Inset: values of $\mathrm{NiFe-ODS}$ and the exfoliated $\mathrm{NiFe-LDH}$ using $\mathrm{Ni}$ foam and glassy carbon rotating disc electrode (RDE). C) Overpotential required for a current density of $10 \mathrm{~mA} \cdot \mathrm{cm}^{-2}$. D) Current density at an overpotential of $0.3 \mathrm{~V}$. E) Turnover frequencies calculated at $0.3 \mathrm{~V}$ overpotential.

derivatives, indicating a clear tendency with a better current response when increasing the potential. This could be related to the fact that the total accessible electroactive sites are superior, leading to higher catalytic activity (vide infra). It is important to note that the electrocatalytic activity was performed with $\mathrm{Ni}$ foam collectors as the working electrode. Interestingly, when using a $3 \mathrm{~mm}$ diameter glassy carbon rotating disc electrode (RDE), Tafel slope reaches values in the range of $23-31 \mathrm{mV} \cdot \mathrm{dec}^{-1}$ surpassing the value of $36 \mathrm{mV} \cdot \mathrm{dec}^{-1}$ previously reported in the literature for the NiFe-DS. ${ }^{14}$. Going further, Tafel slope of an exfoliated NiFe-LDH -which represents the limit case- using the same experimental conditions, ranges between 19-26 mV.dec ${ }^{-1}$. Therefore, NiFe-ODS displays a kinetic response similar to the one exhibited by an exfoliated benchmark LDH (Tafel slope calculations were done from NiFe-ODS and NiFe-exf. LSV data shown in Figure S16).

Overpotential values required for $10 \mathrm{~mA} \cdot \mathrm{cm}^{-2}$ are displayed in Figure ${ }_{4} \mathrm{C}$. This current density was chosen because it represents the current density from a device with $12 \%$ solar to hydrogen efficiency. ${ }^{15}$ Overpotential obtained present an improvement until NiFe-DS sample and then a slight drop tendency. As described by Hunter et al. there is a strong correlation between water oxidation activity and the $\mathrm{pK}_{\mathrm{a}}$ of the conjugate acids of the interlayer anions. ${ }^{13}$ In this sense, the decrease in this value when replacing chloride by sulphate can be attributed to an increase of the $\mathrm{pK}_{\mathrm{a}}$ from $-7(\mathrm{NiFe}-\mathrm{Cl})$ to $1.99(\mathrm{NiFe}-$ $\left.\mathrm{SO}_{4}\right)$. For the sulphate derivatives, the $\mathrm{pK}_{\mathrm{a}}$ value stays rather constant (1.90 for NiFe-DS), so the differences do not seem to come from anion basicity. Figure $4 \mathrm{D}$ reveals the current density measured at a fixed overpotential of o.3 V. Remarkably, a similar trend along the compounds is observed, with a current density maximum in NiFe-DS. To go deeper into the electrocatalytic comparison, TOF values were calculated at an overpotential of $300 \mathrm{mV}$, assuming that all the metal ions in the LDHs were active. Figure $4 \mathrm{E}$ shows that the TOF values follow the same course indicating the best OER at lower overpotentials for the NiFe-DS instead of the NiFe-ODS sample.

To further understand the observed electrochemical behaviour, we extracted the electrochemical surface area (ECSA) of all the LDH-family. Indeed, it is well-known that the increase in the specific surface area and thus, in the number of electroactive sites leads to an improvement 
A)
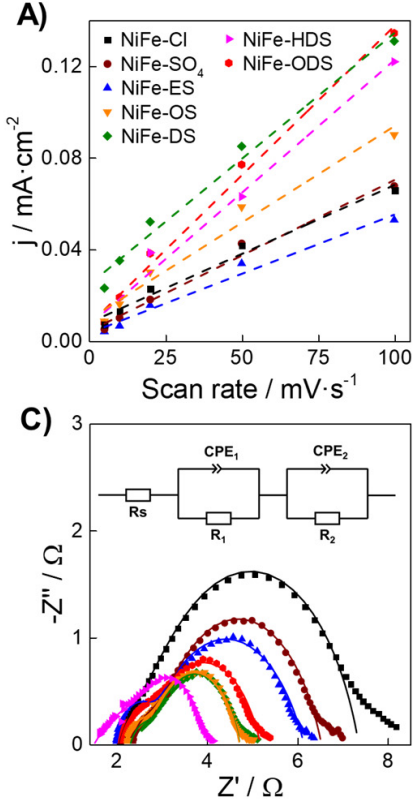

E)
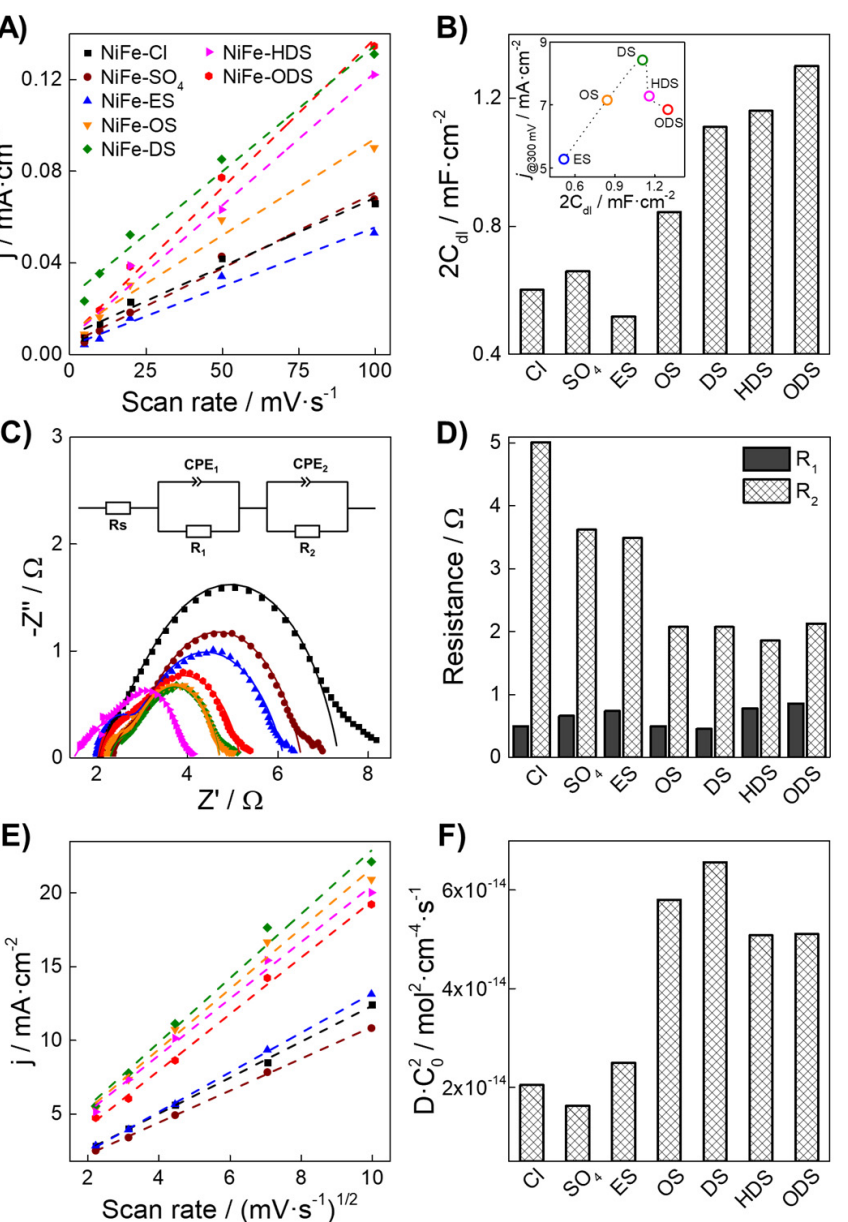

F)

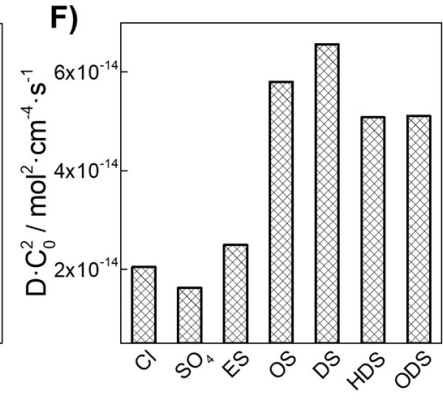

Figure 5. A) Electrochemical surface area (ECSA) of the different samples calculated from CVs performed in a nonfaradaic region at different scan rates. B) Linear slope values representing the ECSA. Inset: Current density at $300 \mathrm{mV}$ overpotential plotted against ECSA for the surfactant intercalated compounds. C) Electrochemical impedance spectra (EIS) of NiFe-LDH samples recorded at an overpotential of $0.3 \mathrm{~V}$. Points correspond to experimental data and line is a curve fitted with the equivalent circuit. D) Resistance values obtained from EIS data. (E) Linear relationship of the current of the peak potentials and scan rates for the different $\mathrm{NiFe}-\mathrm{LDH}$ samples during the oxidation of $\mathrm{Ni}(\mathrm{OH})_{2}$. (F) Values of the diffusion coefficient of the proton multiplied by the proton concentration for the different samples.

of the OER performance. ${ }^{15}$ ECSA of each compound were analysed by obtaining the double layer capacitance (Figure $5 \mathrm{~A}$ ); the slopes of the fitted plots correspond to the double layer capacitance whose values are plotted in Figure $5 \mathrm{~B}$. Remarkably, the ECSA shows a similar trend that the one observed in the Tafel slope, with values going from $0.6 \mathrm{mF} \cdot \mathrm{cm}^{-2}(\mathrm{NiFe}-\mathrm{Cl})$ to $1.3 \mathrm{mF} \cdot \mathrm{cm}^{-2}$ (NiFe-ODS). Therefore, there is a strong correlation between the Tafel slope improvement and the increase of electroactive sites achieved by enlarging the interlayer LDH distance. Regarding the current values, the inset in Figure ${ }_{5} \mathrm{~B}$ displays a linear relation between ECSA and current at $300 \mathrm{mV}$

overpotential for the surfactant samples until NiFe-DS. Enlarging more the interlayer distance leads to higher ECSA and consequently does not explain the decrease of the activity at low overpotentials.

In order to shed light on the observed trend, electrochemical impedance spectroscopy (EIS) which is a useful technique to understand electrocatalytic processes, was performed (Figure ${ }_{5} \mathrm{C}$ ). The equivalent circuit used to fit these data contains a resistance Rs in series with two circuits where $R_{S}$ corresponds to the ionic transport resistance through the solution. The high-frequency arc $\left(\mathrm{R}_{1}\right.$, $\mathrm{CPE}_{1}$ ) could be associated to the charge transfer from the $\mathrm{Ni}$ foam to the electroactive sites, and the low-frequency arc $\left(\mathrm{R}_{2}, \mathrm{CPE}_{2}\right)$ could be related with the chemisorption of oxygen. Resistance values from each frequency arc are shown in Figure ${ }_{5} \mathrm{D} . \mathrm{R}_{2}$ decreases as well as the interlayer distance increases reaching a minimum in NiFe-OS sample that remains similar for higher basal spaces. The reduction of $\mathrm{R}_{2}$ can be associated with a larger number of active centers that are facilitating the oxygen formation (thus, increasing the OER performance). However, the electrocatalytic activity continues improving by enlarging the interlayer space beyond $22.3 \AA$ (NiFe-OS), while $\mathrm{R}_{2}$ does not vary. Therefore, for larger interlayer distances, the OER enhancement is mainly due to the ECSA increment.

The combination of ECSA and EIS suggest that the larger electroactive sites and the decrease of the resistance are vital factors responsible for the advanced electrochemical performance on water oxidation. However, the above discussion explains the Tafel slope progress but does not clarify the optimum behaviour recorded for $\mathrm{NiFe}-\mathrm{DS}$ (Figures ${ }_{4} \mathrm{~B}, 4 \mathrm{C},{ }_{4} \mathrm{E}$ and inset of ${ }_{5} \mathrm{~B}$ ). In that case, we have to focus on the charge transfer from $\mathrm{Ni}$ foam to the electroactive sites. In fact, $\mathrm{R}_{1}$ does not continue a tendency and reaches the lowest value for $\mathrm{NiFe-DS}$. Surprisingly, the highest value corresponds to the NiFe-ODS sample. The increment of this resistance could be responsible for a higher onset value of LDHs with a basal space beyond $\mathrm{NiFe}-\mathrm{DS}$.

The mechanism for OER on NiFe-LDH is still under debate and investigation. ${ }^{9}$ The nickel oxyhydroxide has been investigated as an active phase for $\mathrm{OER}^{45,46}$ and the iron oxyhydroxide possibly plays a crucial role. ${ }^{47}$ Nevertheless, while the iron peak is surely masked because of the OER, the nickel oxidation (considered vital for high OER performance) can be experimentally detected and thus, studied by means of CVs. ${ }^{48-50}$ Keeping this in mind, Luan et al. studied the proton diffusion coefficient during $\mathrm{Ni}(\mathrm{OH})_{2}$ oxidation that can be estimated for diffusionlimited reactions using the Randles-Sevcik equation. ${ }^{51} \mathrm{~A}$ higher proton diffusion coefficient is related to faster oxidation of $\mathrm{Ni}(\mathrm{OH})_{2}$ to $\mathrm{NiOOH}$ phase $(\mathrm{NiFeOOH}$ in our case) and then it leads to an improvement on the overpotentials required for starting the OER. From CVs (Figure S13), NiFe-LDH oxidation peak currents were plotted against the square root of the scan rate obtaining a linear 


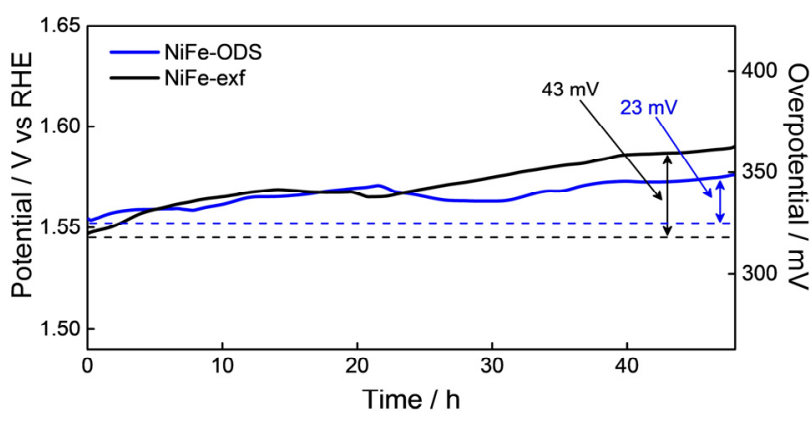

Figure 6. Stability of NiFe-ODS and NiFe-exfoliated under a constant current density of $10 \mathrm{~mA} \cdot \mathrm{cm}^{-2}$ during 48 hours. The overpotential required is increased by $23 \mathrm{mV}$ and $43 \mathrm{mV}$ for the NiFe-ODS and the NiFe-exf respectively, showing the better performance of the tridimensional material.

relationship (Figure $5 \mathrm{E}$ ). Therefore, the Randles-Sevcik equation can be used to extract and compare the proton diffusion coefficient values of all NiFe-LDH samples (Figure ${ }_{5} \mathrm{~F}$ ). These results clearly point towards an improvement of the proton diffusion, reaching a maximum for $\mathrm{NiFe-DS}$, in excellent agreement with the trends observed in Figures ${ }_{4} \mathrm{C}$ and ${ }_{4} \mathrm{D}$. Along this front, the higher number of carbons in the surfactants results in an increment of the hydrophobicity of the interlayer space as well as in stronger hydrophobic interactions among the longerchain sulphates. Thus the proton diffusion from the hydroxide layer to the solution is reduced. ${ }^{52-54}$ In this manner, the increase of the basal space leads to a better proton diffusion (therefore, to faster oxidation) until reaching a distance where the hydrophobic contribution becomes significant. This suggests that the ratio between hydroxide/oxyhydroxide is higher in NiFe-HDS and $\mathrm{NiFe-ODS}$ compared to NiFe-DS. It is important to remark the fact that $\mathrm{NiFe}-\mathrm{LDH}$ is an insulator whereas $\mathrm{NiFeOOH}$ is considered as a semiconductor. ${ }^{9,55,56}$ Thus, the superior hydroxide/oxyhydroxide ratio could be responsible for the increase in $R_{1}$ for an interlayer distance beyond NiFe-DS. It is worth to remark that this is a complex process as mentioned above, where structural changes and ion dynamics may also play a key role in the electrochemical behaviour. ${ }^{57}$

Last but not least, stability and durability of the more and less spaced LDHs ( $\mathrm{NiFe-ODS}$ and $\mathrm{NiFe}-\mathrm{Cl}$, respectively) were tested at constant current densities of 5 and 10 $\mathrm{mA} \cdot \mathrm{cm}^{-2}$ and at constant overpotentials of $300 \mathrm{mV}$ and $400 \mathrm{mV}$ for $2 \mathrm{~h}$. High stability is observed in all conditions (Figure S17). Moreover, in order to demonstrate the usefulness of the anion exchange approach, the stability during $48 \mathrm{~h}$ under a current density of $10 \mathrm{~mA} \cdot \mathrm{cm}^{-2}$ was recorded for the $\mathrm{NiFe}-\mathrm{ODS}$ versus the exfoliated $\mathrm{NiFe}-$ LDH (Figure 6). The variation of the required overpotential after this long-time experiment shows an increase of ca. $7 \%$ and $13 \%$ for the NiFe-ODS and the exfoliated $\mathrm{LDH}$, respectively. Therefore, the NiFe-ODS exhibits a similar kinetic behaviour that the exfoliated LDH while presenting a bigger structural resilience due to its tridimensional structure, opening the door to the design of improved catalytic materials.

Given the high tendency of LDH phases towards anion exchange reactions to incorporate carbonates, ${ }^{57}$ we have checked by ATR-FTIR that the surfactant molecules remain intercalated during the different electrochemical tests (Figure S18). Only for the longer stability tests it is possible to appreciate that the intensity of the characteristic surfactant peaks decreases concomitantly with the appearance of the carbonate peak located at ca. $1360 \mathrm{~cm}^{-1}$.

\section{CONCLUSIONS}

In summary, we have reported the synthesis by anion exchange reactions of a family of surfactant-intercalated $\mathrm{NiFe}-\mathrm{LDH}$ having similar crystallinity, metal composition, morphology and dimensions, but endowed with different interlayer anions. This has allowed us to exclusively vary in this LDH family the basal space. The oxygen evolution reaction was explored to analyse the impact of interlayer distance on the electrocatalytic performance. The increment of the basal space results in a decrease of the Tafel slopes, an increase of the electrochemical surface area and a reduction of the resistance connected to the oxygen chemisorption that improves the kinetic behaviour. Interestingly, we observed an optimum onset potential for the NiFe-DS sample (basal space of 25.2 $\AA$ ). A further increase in the basal space compromises the onset potential likely due to proton diffusion limitations as a consequence of the pronounced alkyl-chain hydrophobicity between the layers. Finally, by maximizing the basal space to $31.6 \AA$, a very small Tafel slope of ca. 27 $\mathrm{mV} \cdot \mathrm{dec}^{-1}$, similar to that of exfoliated $\mathrm{NiFe}-\mathrm{LDHs}$, can be achieved, but showing a much better long-term stability as a consequence of the tridimensional robustness of the hybrid material. This work provides significant insights into the OER activity of NiFe-LDHs, illustrating the importance of molecular engineering in the design of novel high-performance catalytic materials.

\section{ASSOCIATED CONTENT}

The Supporting Information is available free of charge via the Internet at http://pubs.acs.org.

X-Ray Diffraction, Infrared spectra, Thermogravimetric analysis, DLS measurements, AFM measurements, SEM, TEM, XPS spectra, Molecular formula, Porosity measurements, and additional electrochemical measurements.

\section{AUTHOR INFORMATION}

Corresponding Author

* G. Abellán. E-mail: gonzalo.abellan@uv.es

* E. Coronado. E-mail: eugenio.coronado@uv.es

Author Contributions

J.A.C and R.S-G contributed equally. 
Notes

The authors declare no competing financial interest.

\section{ACKNOWLEDGMENT}

This work was partially supported by the European Research Council (ERC Advanced Grant Mol-2D 788222 to E.C. and ERC Starting Grant 2D-PnictoChem 804110 to G.A.). G.A. thanks support by the Deutsche Forschungsgemeinschaft (DFG; FLAG-ERA AB694/2-1), and the Generalitat Valenciana (SEJI/2018/034 grant and CIDEGENT/2018/oo1). R.S.-G. thanks the Ministerio de Ciencia, Innovación y Universidades for the F.P.U. fellowship. This work was supported by the Spanish MINECO (Unit of Excellence "Maria de Maeztu" MDM-2015-0538 and Project MAT2017-89993-R co-financed by FEDER), and Generalitat Valenciana (Prometeo/2017/o66).

\section{REFERENCES}

(1) Jin, H.; Guo, C.; Liu, X.; Liu, J.; Vasileff, A.; Jiao, Y.; Zheng, Y.; Qiao, S.-Z. Emerging Two-Dimensional Nanomaterials for Electrocatalysis. Chem. Rev. 2018, 118 (13), 6337-6408.

(2) Shao, M.; Zhang, R.; Li, Z.; Wei, M.; Evans, D. G.; Duan, X. Layered Double Hydroxides toward Electrochemical Energy Storage and Conversion: Design, Synthesis and Applications. Chem. Commun. 2015, 51 (88), 15880-15893.

(3) Zhao, M.; Zhao, Q.; Li, B.; Xue, H.; Pang, H.; Chen, C. Recent Progress in Layered Double Hydroxide Based Materials for Electrochemical Capacitors: Design, Synthesis and Performance. Nanoscale 2017, 9 (40), 1520615225 .

(4) Wang, Q.; O'Hare, D. Recent Advances in the Synthesis and Application of Layered Double Hydroxide (LDH) Nanosheets. Chem. Rev. 2012, 112 (7), 4124-4155.

(5) Yu, J.; Wang, Q.; O'Hare, D.; Sun, L. Preparation of Two Dimensional Layered Double Hydroxide Nanosheets and Their Applications. Chem. Soc. Rev. 2017, 46 (19), 5950-5974.

(6) Fan, G.; Li, F.; Evans, D. G.; Duan, X. Catalytic Applications of Layered Double Hydroxides: Recent Advances and Perspectives. Chem. Soc. Rev. 2014, 43 (20), 70407066.

(7) Tian, R.; Liang, R.; Wei, M.; Evans, D. G.; Duan, X. Applications of Layered Double Hydroxide Materials: Recent Advances and Perspective; Springer Berlin Heidelberg: Berlin, Heidelberg, 2015.

(8) Abellán, G.; Martí-Gastaldo, C.; Ribera, A.; Coronado, E. Hybrid Materials Based on Magnetic Layered Double Hydroxides: A Molecular Perspective. Acc. Chem. Res. 2015, 48 (6), 1601-1611.

(9) Dionigi, F.; Strasser, P. NiFe-Based (Oxy)Hydroxide Catalysts for Oxygen Evolution Reaction in Non-Acidic Electrolytes. Adv. Energy Mater. 2016, 6 (23), 1600621.

(10) Abellán, G.; Carrasco, J. A.; Coronado, E.; Romero, J.; Varela, M. Alkoxide-Intercalated CoFe-Layered Double Hydroxides as Precursors of Colloidal Nanosheet Suspensions: Structural, Magnetic and Electrochemical Properties. J. Mater. Chem. C 2014, 2 (19), 3723-3731.

(11) Zhao, Y.; Zhang, X.; Jia, X.; Waterhouse, G. I. N.; Shi, R.; Zhang, X.; Zhan, F.; Tao, Y.; Wu, L.-Z.; Tung, C.-H.; et al. Sub-3 Nm Ultrafine Monolayer Layered Double Hydroxide Nanosheets for Electrochemical Water Oxidation. Adv. Energy Mater. 2018, 8 (18), 1703585.

(12) Carrasco, J. A.; Harvey, A.; Hanlon, D.; Lloret, V.; McAteer, D.; Sanchis-Gual, R.; Hirsch, A.; Hauke, F.; Abellán, G.; Coleman, J. N.; et al. Liquid Phase Exfoliation of Carbonate-Intercalated Layered Double Hydroxides. Chem. Commun. 2019, 55 (23), 3315-3318.

(13) Hunter, B. M.; Hieringer, W.; Winkler, J. R.; Gray, H. B.; Müller, A. M. Effect of Interlayer Anions on [NiFe]LDH Nanosheet Water Oxidation Activity. Energy Environ. Sci. 2016, 9 (5), 1734-1743.

(14) Dang, L.; Liang, H.; Zhuo, J.; Lamb, B. K.; Sheng, H.; Yang, Y.; Jin, S. Direct Synthesis and Anion Exchange of Noncarbonate-Intercalated NiFe-Layered Double Hydroxides and the Influence on Electrocatalysis. Chem. Mater. 2018, 30 (13), 4321-4330.

(15) Song, F.; Hu, X. Exfoliation of Layered Double Hydroxides for Enhanced Oxygen Evolution Catalysis. Nat. Commun. 2014, 5 (4477), 1-9.

(16) Abellán, G.; Coronado, E.; Martí-Gastaldo, C.; PinillaCienfuegos, E.; Ribera, A. Hexagonal Nanosheets from the Exfoliation of Ni2+-Fe3+ LDHs: A Route towards Layered Multifunctional Materials. J. Mater. Chem. 2010, 20 (35), 7451-7455.

(17) Abellán, G.; Carrasco, J. A.; Coronado, E. Room Temperature Magnetism in Layered Double Hydroxides Due to Magnetic Nanoparticles. Inorg. Chem. 2013, 52 (14), 7828-7830.

(18) Carrasco, J. A.; Cardona-Serra, S.; Clemente-Juan, J. M.; Gaita-Ariño, A.; Abellán, G.; Coronado, E. Deciphering the Role of Dipolar Interactions in Magnetic Layered Double Hydroxides. Inorg. Chem. 2018, 57 (4), 20132022.

(19) Abellán, G.; Coronado, E.; Gómez-García, C. J.; MartíGastaldo, C.; Ribera, A. Intercalation of Cobalt(II)Tetraphenylporphine Tetrasulfonate Complex in Magnetic NiFe-Layered Double Hydroxide. Polyhedron 2013, 52, 216-221.

(20) Abellán, G.; Coronado, E.; Martí-Gastaldo, C.; Waerenborgh, J.; Ribera, A. Interplay between Chemical Composition and Cation Ordering in the Magnetism of $\mathrm{Ni} / \mathrm{Fe}$ Layered Double Hydroxides. Inorg. Chem. 2013, 52 (17), 10147-10157.

(21) Horcas, I.; Fernández, R.; Gómez-Rodríguez, J. M.; Colchero, J.; Gómez-Herrero, J.; Baro, A. M. WSXM: A Software for Scanning Probe Microscopy and a Tool for Nanotechnology. Rev. Sci. Instrum. 2007, 78 (1), 013705-013708.

(22) Carrasco, J. A.; Abellán, G.; Coronado, E. Influence of Morphology in the Magnetic Properties of Layered Double Hydroxides. J. Mater. Chem. C 2018, 6 (5), 11871198.

(23) Zhou, L.-J.; Huang, X.; Chen, H.; Jin, P.; Li, G.-D.; Zou, X. A High Surface Area Flower-like Ni-Fe Layered Double Hydroxide for Electrocatalytic Water Oxidation Reaction. Dalton Trans. 2015, 44 (25), 11592-116oo.

(24) Viana, R. B.; da Silva, A. B. F.; Pimentel, A. S. Infrared Spectroscopy of Anionic, Cationic, and Zwitterionic Surfactants. Adv. Phys. Chem. 2012, 2012, 1-14.

(25) Liu, Y.; Yang, Z. Intercalation of Sulfate Anions into a Zn-Al Layered Double Hydroxide: Their Synthesis and 
Application in Zn-Ni Secondary Batteries. RSC Adv. 2016, 6 (73), 68584-68591.

(26) Meyn, M.; Beneke, K.; Lagaly, G. Anion-Exchange Reactions of Hydroxy Double Salts. Inorg. Chem. 1993, 32 (7), 1209-1215.

(27) Carrasco, J. A.; Romero, J.; Varela, M.; Hauke, F.; Abellán, G.; Hirsch, A.; Coronado, E. Alkoxide-Intercalated NiFe-Layered Double Hydroxides Magnetic Nanosheets as Efficient Water Oxidation Electrocatalysts. Inorg. Chem. Front. 2016, 3 (4), 478-487.

(28) Zhang, J.; Xie, X.; Li, C.; Wang, H.; Wang, L. The Role of Soft Colloidal Templates in the Shape Evolution of Flower-like MgAl-LDH Hierarchical Microstructures. RSC Adv. 2015, 5 (38), 29757-29765.

(29) Theiss, F. L.; Ayoko, G. A.; Frost, R. L. Thermogravimetric Analysis of Selected Layered Double Hydroxides. J. Therm. Anal. Calorim. 2o13, 112 (2), 649-657.

(30) Gardner, E.; Huntoon, K. M.; Pinnavaia, T. J. Direct Synthesis of Alkoxide-Intercalated Derivatives of Hydrocalcite-like Layered Double Hydroxides: Precursors for the Formation of Colloidal Layered Double Hydroxide Suspensions and Transparent Thin Films. Adv. Mater. 2001, 13 (16), 1263-1266.

(31) Gursky, J. A.; Blough, S. D.; Luna, C.; Gomez, C.; Luevano, A. N.; Gardner, E. A. Particle-Particle Interactions between Layered Double Hydroxide Nanoparticles. J. Am. Chem. Soc. 2oo6, 128 (26), 8376-8377.

(32) Constantino, V. R.; Pinnavaia, T. J. Basic Properties of $\mathrm{Mg}^{2+}{ }_{1-\mathrm{X}} \mathrm{Al}^{3+}{ }_{\mathrm{x}}$ Layered Double Hydroxides Intercalated by Carbonate, Hydroxide, Chloride, and Sulfate Anions. Inorg. Chem. 1995, 34 (4), 883-892.

(33) Yun, S. K.; Pinnavaia, T. J. Water Content and Particle Texture of Synthetic Hydrotalcite-like Layered Double Hydroxides. Chem. Mater. 1995, 7 (2), 348-354.

(34) Abellán, G.; Coronado, E.; Martí-Gastaldo, C.; Ribera, A.; Jordá, J. L.; García, H. Photo-Switching in a Hybrid Material Made of Magnetic Layered Double Hydroxides Intercalated with Azobenzene Molecules. Adv. Mater. 2014, 26 (24), 4156-4162.

(35) Raki, L.; Rancourt, D. G.; Detellier, C. Preparation, Characterization, and Moessbauer Spectroscopy of Organic Anion Intercalated Pyroaurite-like Layered Double Hydroxides. Chem. Mater. 1995, 7 (1), 221-224.

(36) Barbosa, C. A. S.; Ferreira, A. M. D. C.; Constantino, V. R. L.; Coelho, A. C. V. Preparation and Characterization of $\mathrm{Cu}(\mathrm{II})$ Phthalocyanine Tetrasulfonate Intercalated and Supported on Layered Double Hydroxides. J. Incl. Phenom. Macrocycl. Chem. 2002, 42 (1-2), 15-23.

(37) Juang, L.-C.; Lee, C.-K.; Wang, C.-C.; Hung, S.-H.; Lyu, M.-D. Adsorptive Removal of Acid Red 1 from Aqueous Solution with Surfactant Modified Titanate Nanotubes. Environ. Eng. Sci. 20o8, 25 (4), 519-528.

(38) Zhang, Y.; Zhao, Y.; Zhu, Y.; Wu, H.; Wang, H.; Lu, W. Adsorption of Mixed Cationic-Nonionic Surfactant and Its Effect on Bentonite Structure. J. Environ. Sci. 2012, 24 (8), 1525-1532.

(39) Romero, J.; Rodriguez-San-Miguel, D.; Ribera, A.; MasBallesté, R.; Otero, T. F.; Manet, I.; Licio, F.; Abellán, G.; Zamora, F.; Coronado, E. Metal-Functionalized Covalent Organic Frameworks as Precursors of Supercapacitive Porous N-Doped Graphene. J. Mater. Chem. A 2017, 5 (9), 4343-4351.
(40) Marcus, Y. Volumes of Aqueous Hydrogen and Hydroxide Ions at o to $200{ }^{\circ} \mathrm{C}$. J. Chem. Phys. 2012, 137 (15), 154501.

(41) Liu, Z.; Ma, R.; Osada, M.; Iyi, N.; Ebina, Y.; Takada, K.; Sasaki, T. Synthesis, Anion Exchange, and Delamination of Co-Al Layered Double Hydroxide: Assembly of the Exfoliated Nanosheet/Polyanion Composite Films and Magneto-Optical Studies. J. Am. Chem. Soc. 2oo6, 128 (14), 4872-488o.

(42) Yan, K.; Lafleur, T.; Chai, J.; Jarvis, C. Facile Synthesis of Thin NiFe-Layered Double Hydroxides Nanosheets Efficient for Oxygen Evolution. Electrochem. Commun 2016, 62, 24-28.

(43) Gong, M.; Li, Y.; Wang, H.; Liang, Y.; Wu, J. Z.; Zhou, J.; Wang, J.; Regier, T.; Wei, F.; Dai, H. An Advanced Ni-Fe Layered Double Hydroxide Electrocatalyst for Water Oxidation. J. Am. Chem. Soc. 2013, 135 (23) $845^{-}-8455$.

(44) Biesinger, M. C.; Payne, B. P.; Grosvenor, A. P.; Lau, L. W. M.; Gerson, A. R.; Smart, R. St. C. Resolving Surface Chemical States in XPS Analysis of First Row Transition Metals, Oxides and Hydroxides: $\mathrm{Cr}, \mathrm{Mn}, \mathrm{Fe}, \mathrm{Co}$ and Ni. Appl. Surf. Sci. 2011, 257 (7), 2717-2730.

(45) Lu, Z.; Xu, W.; Zhu, W.; Yang, Q.; Lei, X.; Liu, J.; Li, Y.; Sun, X.; Duan, X. Three-Dimensional NiFe Layered Double Hydroxide Film for High-Efficiency Oxygen Evolution Reaction. Chem. Commun. 2014, 50 (49), 6479-6482.

(46) Zhang, J.; Liu, J.; Xi, L.; Yu, Y.; Chen, N.; Sun, S.; Wang, W.; Lange, K. M.; Zhang, B. Single-Atom Au/NiFe Layered Double Hydroxide Electrocatalyst: Probing the Origin of Activity for Oxygen Evolution Reaction. J. Am. Chem. Soc. 2018, 140 (11), 3876-3879.

(47) Enman, L. J.; Stevens, M. B.; Dahan, M. H.; Nellist, M. R.; Toroker, M. C.; Boettcher, S. W. Operando X-Ray Absorption Spectroscopy Shows Iron Oxidation Is Concurrent with Oxygen Evolution in Cobalt-Iron (Oxy)Hydroxide Electrocatalysts. Angew. Chem. Int. Ed. 2018, 57 (39), 12840-12844.

(48) Bediako, D. K.; Lassalle-Kaiser, B.; Surendranath, Y.; Yano, J.; Yachandra, V. K.; Nocera, D. G. StructureActivity Correlations in a Nickel-Borate Oxygen Evolution Catalyst. J. Am. Chem. Soc. 2012, 134 (15), 68016809.

(49) Li, Y.-F.; Selloni, A. Mechanism and Activity of Water Oxidation on Selected Surfaces of Pure and Fe-Doped $\mathrm{NiO}_{x}$. ACS Catal. 2014, 4 (4), 1148-1153.

(50) Lu, P. W. T. Electrochemical-Ellipsometric Studies of Oxide Film Formed on Nickel during Oxygen Evolution. J. Electrochem. Soc. 1978, 125 (9), 1416.

(51) Luan, C.; Liu, G.; Liu, Y.; Yu, L.; Wang, Y.; Xiao, Y.; Qiao, H.; Dai, X.; Zhang, X. Structure Effects of 2D Materials on $\alpha$-Nickel Hydroxide for Oxygen Evolution Reaction. ACS Nano 2018, 12 (4), 3875-3885.

(52) Zhang, H.; Xu, Z. P.; Lu, G. Q.; Smith, S. C. Intercalation of Sulfonate into Layered Double Hydroxide: Comparison of Simulation with Experiment. J. Phys. Chem. C 2009, 113 (2), 559-566.

(53) Chen, L.-J.; Lin, S.-Y.; Huang, C.-C. Effect of Hydrophobic Chain Length of Surfactants on Enthalpy-Entropy Compensation of Micellization. J. Phys. Chem. B 1998, 102 (22), 4350-4356. 
(54) Xu, Z. P.; Braterman, P. S. Competitive Intercalation of Sulfonates into Layered Double Hydroxides (LDHs): The Key Role of Hydrophobic Interactions. J. Phys. Chem. C 2007, 111 (10), 4021-4026.

(55) Motori, A.; Sandrolini, F.; Davolio, G. Electrical Properties of Nickel Hydroxide for Alkaline Cell Systems. J. Power Sources 1994, 48 (3), 361-370.

(56) Trotochaud, L.; Young, S. L.; Ranney, J. K.; Boettcher, S. W. Nickel-Iron Oxyhydroxide Oxygen-Evolution Electrocatalysts: The Role of Intentional and Incidental Iron Incorporation. J. Am. Chem. Soc. 2014, 136 (18), 6744-6753.

(57) Taviot-Guého, C.; Vialat, P.; Leroux, F.; Razzaghi, F.; Perrot, H.; Sel, O.; Jensen, N. D.; Nielsen, U. G.; Peulon, S.; Elkaim, E.; et al. Dynamic Characterization of Inter- and Intralamellar Domains of Cobalt-Based Layered Double Hydroxides upon Electrochemical Oxidation. Chem. Mater. 2016, 28 (21), 7793-7806. 


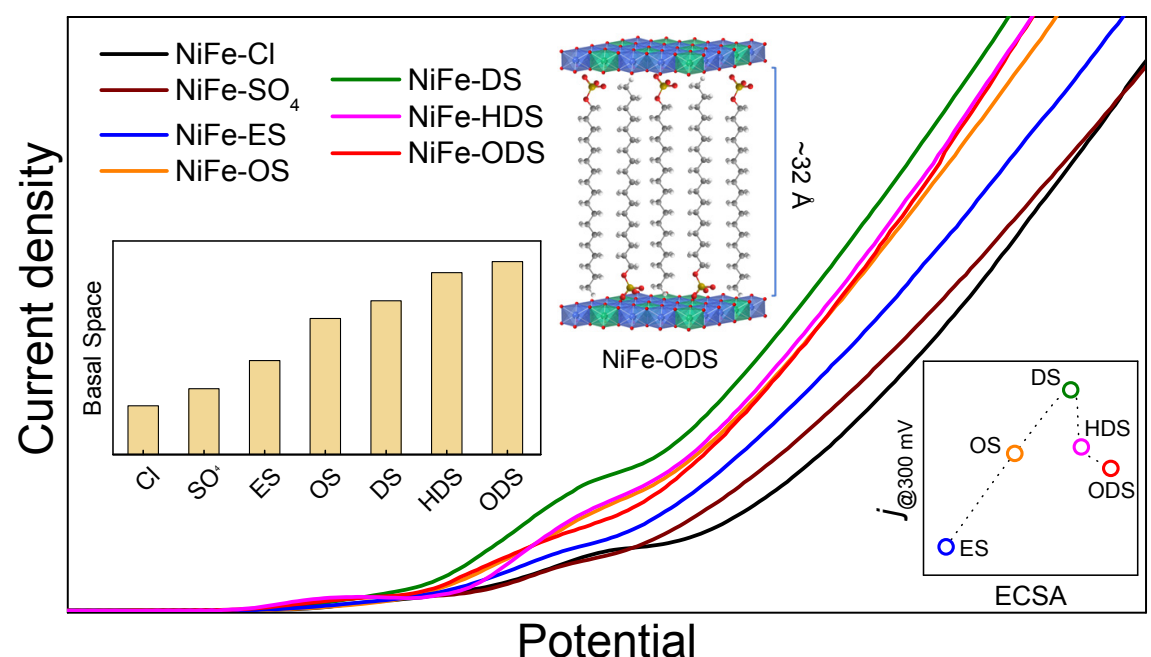

